\title{
Tentativa de suicídio e fatores associados ao padrão uso e abuso do álcool
}

\author{
Eliana Lessa Cordeiro ${ }^{1}$ \\ (D) https://orcid.org/0000-0001-7305-9431 \\ Liniker Scolfild Rodrigues da Silva² \\ (D) https://orcid.org/0000-0003-3710-851X \\ Emmanuel Wagner Pereira Mendes ${ }^{3}$ \\ (D) https://orcid.org/0000-0002-2936-1614 \\ Luiz Claudio Luna da Silva ${ }^{3}$ \\ (D) https://orcid.org/0000-0001-9815-6588 \\ Vanessa Lacerda Duarte ${ }^{3}$ \\ (D) https://orcid.org/0000-0001-6873-3508 \\ Évelyn Cristina Morais Pessôa Lima \\ (D) https://orcid.org/0000-0002-1862-8491
}

1 Universidade Federal de Pernambuco, Cidade Universitária, Recife, PE, Brasil.

2 Universidade de Pernambuco, Faculdade de Ciências Médicas, Recife, PE, Brasil.

${ }^{3}$ Universidade Salgado de Oliveira, Recife, PE, Brasil.

${ }^{4}$ Universidade de Pernambuco, Faculdade Nossa Senhora das Graças, Recife, PE, Brasil.
Objetivo: este estudo teve como objetivo relacionar o padrão de uso, abuso e problemas relacionados ao álcool entre os pacientes que tentaram suicídio em Recife no ano de 2015. Método: trata-se de um estudo transversal, prospectivo, do tipo descritivo, epidemiológico, de abordagem quantitativa, no município do Recife-PE, realizado nas residências dos pacientes que tentaram suicídio e cujo nome consta em notificações compulsórias enviadas para a Vigilância de Atenção a Doenças não Transmissíveis da Secretaria Municipal do Recife. Para a coleta dos dados, aplicou-se o teste AUDIT, por meio de entrevistas, sendo esses dados organizados com o programa EPI-7 e analisados no programa SPSS $^{\circledR}$, versão 21.0. Resultados: apresentou-se uma prevalência maior em mulheres jovens, com idade média de 38 anos, com $1^{\circ}$ grau de escolaridade, cor parda, solteiras, desempregadas e pertencentes à classe social C. No teste AUDIT, 41,4\% dos entrevistados encontram-se na zona I, necessitando de aconselhamento aos riscos do consumo de álcool, e 24,1\% dos pacientes bebem. Conclusão: os comportamentos suicidas acentuam-se com o uso abusivo do álcool e, por isso, devem ser encarados pelas equipes de saúde com seriedade, respeito e compromisso, pois os agravos e as consequências dessas complicações se tornam ainda mais difíceis quando negligenciados.

Descritores: Suicídio; Tentativas; Álcool; Alcoolismo.

\section{Como citar este artigo}

Cordeiro EL, Silva LSR, Mendes EWP, Silva LCL, Duarte VL, Lima ECMP. Suicide attempt and factors associated with standard alcohol use and abuse. SMAD, Rev Eletrônica Saúde Mental Álcool Drog. 2020;16(1):1-10. doi: https://dx.doi.org/10.11606/issn.1806-6976.smad.2020.157007 


\title{
Suicide attempt and factors associated with standard alcohol use and abuse
}

\begin{abstract}
Objective: the present study aimed to relate the pattern of abuse and alcohol-related problems among patients who attempted suicide in Recife in the year 2015. Method: this is a cross-sectional, prospective, descriptive, epidemiological type of quantitative approach in the municipality of Recife-PE, carried out in the residences of patients who attempted suicide and whose name appears in compulsory notifications, sent to noncommunicable surveillance of the Municipal Secretariat of Recife. For the data collection, the AUDIT test was applied through interviews, having these data organized with the EPI-7 program and analyzed in the SPSS ${ }^{\circledR}$ program, version 21.0. Results: a higher prevalence was found in young women, with a mean age of 38 years, with a first degree of education, brown, single, unemployed and social class C. In the AUDIT test $41.4 \%$ of the interviewees are in the area I, requiring advice on the risks of alcohol consumption and $24.1 \%$ of patients who drink. Conclusion: suicidal behaviors are accentuated by the abusive and continuous use of alcohol and therefore should be viewed seriously by the health teams as serious, respectful and committed, as the aggravations and consequences of these complications become even more difficult when neglected.
\end{abstract}

Descriptors: Suicid; Attempts; Alcohol; Alcoholism.

\section{Intento de suicidio y factores asociados al patrón uso y abuso del alcohol}

Objetivo: el presente estudio tuvo como objetivo relacionar el patrón de uso abusivo y problemas relacionados con el alcohol entre pacientes que intentaron suicidarse en Recife en 2015. Método: este es un estudio transversal, prospectivo, del tipo descriptivo, epidemiológico de abordaje cuantitativo en el municipio de Recife-PE, realizado en las residencias de los pacientes que intentaron suicidio, con nombres em notificaciones obligatorias, enviadas para Vigilancia de Atención a Enfermedad no Transmisibles de la Secretaría Municipal de Recife. Para coletar los datos, se aplicó la prueba AUDIT, por entrevistas, organizando los datos en el programa EPI-7 y analizando en el programa $\mathrm{SPSS}^{\circledR}$, versión 21.0. Resultados: se presentó una prevalencia mayor en mujeres jóvenes, con edad media de 38 años, con 1 grado de escolaridad, pardas, solteras, desempleadas y pertecentes a la clase social C. En la prueba AUDIT 41,4\% de los entrevistados se encuentran en la zona I, necesitando asesoramiento a los riesgos del consumo de alcohol y $24,1 \%$ de los pacientes que beben. Conclusion: los comportamientos suicidas se acentuan con el uso abusivo del alcohol y deben ser encarados por los equipos de salud con seriedad y compromiso, pues las consecuencias de esas complicaciones se vuelven más difíciles cuando descuidadas.

Descriptores: Suicidio; Intentos; El alcohol; Alcoholismo. 


\section{Introdução}

$\mathrm{Na}$ atualidade, o suicídio tem sido compreendido como um fenômeno de grande incidência mundial que afeta todas as classes sociais, idades e gêneros. Embora o Brasil apresente um coeficiente baixo de mortalidade por suicídios, por ser um país de dimensões continentais e possuir uma vasta população, o crescimento deste fator é constante, com elevação de cerca de $21 \%$ nos últimos anos, na faixa etária entre 20 e 34 anos $^{(1)}$. Em 2016, a taxa, no Brasil, chegou a 5,8 por 100 mil habitantes, com a notificação de 11.433 mortes por essa causa. O Sistema de Informações sobre Mortalidade (SIM) registrou, entre 2007 e 2016, 106.374 óbitos por suicídio(2).

As associações entre o suicídio e os fatores socioeconômicos e demográficos podem levar a transtornos psicológicos graves. Esses fatores, como ser do sexo masculino; estar na faixa etária entre 15 e 35 anos ou acima dos 75 anos; residir em áreas urbanas; estar aposentado ou desempregado ou fazer uso de alguma droga, podem gerar níveis altos de ansiedade e estresse, o que pode levar o indivíduo a situações de risco psicológico(1).

Assim, o consumo abusivo e contínuo do álcool pode entrar nas estatísticas de suicídio, na concretização do ato ou nas inúmeras tentativas de executá-lo(3).

O álcool é utilizado na busca do prazer momentâneo, diminuição das tensões do dia a dia, ansiedade ou, até mesmo, no alívio das dores físicas. O problema encontra-se no uso desta substância de livre comércio no Brasil, cada vez mais abusivo e frequente, podendo levar o indivíduo a uma dependência, tanto psicológica quanto fisiológica(4).

Diante desse contexto, quanto maior o conhecimento acerca dos riscos de suicídio, maiores as chances e prevenção. Desde 2006, o Brasil vem desenvolvendo estratégias nacionais de prevenção, envolvendo várias atividades, em diferentes níveis, com a qualificação permanente das equipes de saúde. Várias doenças mentais associam-se ao suicídio e à detecção precoce e o tratamento apropriado dessas condições é importante na sua prevenção(5).

Assim, este estudo buscou relacionar o padrão de uso, abuso e problemas relacionados ao álcool entre os pacientes que tentaram suicídio em Recife no ano de 2015, descrevendo o perfil socioeconômico destes, investigando a periocidade do consumo e o tipo de droga utilizada e verificando o padrão de uso e problemas relacionados ao álcool entre pacientes com tentativa de suicídio.

\section{Método}

Trata-se de um estudo primário quanto à originalidade dos dados; observacional quanto à interferência dos pesquisadores sobre o fenômeno estudado; transversal quanto ao período de andamento do estudo; prospectivo quanto à direcionalidade temporal; do tipo descritivo quanto ao perfil de avaliação epidemiológica; não controlado; de prevalência segundo o tipo de frequência; tipo unicêntrico quanto ao levantamento de dados; não aleatório e aberto quanto ao mascaramento no município do Recife-PE.

As pesquisas foram realizadas nas residências dos pacientes que tentaram suicídio, em áreas restritas às adstritas ao município de Recife e cobertas pelas Unidades de Saúde da Família (USF) ou pelo Programa de Agentes Comunitários de Saúde (PACS). Essa opção foi feita para conferir menor sofrimento aos pacientes que tentaram suicídio, por meio do contato com os pesquisadores, intermediado por um (a) agente comunitário de saúde (ACS) com quem o paciente está habituado. Este estudo compreende o período de abril de 2016 a abril de 2017.

No que se refere à população e amostra do estudo, os dados foram registrados por meio das notificações compulsórias de uma população de 374 casos de tentativas de suicídios na região metropolitana do Recife. Excluíram-se, seguindo os critérios de inclusão e exclusão, 283 pacientes, pois 19 não tinham registros do endereço na ficha de notificação; 211 endereços não eram compatíveis com os descritos na ficha de notificação entregue à Vigilância a Saúde; 20 negaram sua participação; um foi a óbito antes da coleta dos dados, sendo entrevistados, portanto, 90 pacientes que tentaram suicídio em Recife, maiores de 18 anos, que aceitaram participar e se enquadraram nos critérios estabelecidos, compondo a amostra da pesquisa.

Os critérios de inclusão foram baseados nos pacientes com suicídio notificado no período de novembro de 2014 a outubro de 2015, com idade de 18 anos ou mais, que tenham residência no município do Recife e concordaram em participar da pesquisa, sendo excluídos pacientes que tenham ido a óbito antes da coleta dos dados, bem como aqueles que mantinham condições de saúde incompatíveis com sua participação ou cujos endereços apontados nas fichas de notificação não identifiquem o paciente que tentou suicídio.

Para a coleta dos dados, foi aplicado o instrumento de caracterização da ingestão alcoólica, sendo empregados dois instrumentos de coleta: parte do questionário Genacis e o teste de identificação de desordens devido ao uso de álcool, identificado pela sigla AUDIT. 
Do questionário Genacis, foram empregadas as questões que possibilitam calcular o uso do álcool (Bloco E) graduado quanto à frequência e quantidade convertidas em risco para a saúde.

Para caracterizar a presença de problemas atribuíveis ao uso excessivo de álcool, foi empregado o teste AUDIT, que se constitui no instrumento indicado pela Organização Mundial de Saúde (OMS) para tal investigação. A caracterização da ingestão de bebida alcoólica foi feita pela soma dos pontos obtidos nas dez perguntas que compõem o teste, a qual possibilita a classificação do indivíduo em uma das quatro zonas de risco para a saúde atribuível ao consumo de álcool(6).

A pontuação, variando de zero a sete pontos, requer exclusivamente aconselhamento quanto aos riscos do consumo de álcool. Quando a pontuação varia entre oito e quinze pontos, o indivíduo é classificado na zona II de risco, a qual exige vigilância e aconselhamento frequentes. Se a ingestão de bebidas alcoólica é ainda maior e o somatório de pontos do AUDIT varia entre 16 a 19 pontos, o indivíduo pertence à zona III de risco, exigindo aconselhamento frequente, monitorização das funções hepáticas e agendamentos mais assíduos de

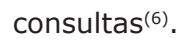

Todavia, se a pontuação iguala-se ou ultrapassa 20 pontos, o indivíduo é classificado como zona IV, ou seja, com necessidade de referenciamento a um especialista para tratamento contra o alcoolismo(6). Os dados foram organizados com o programa EPI-7, disponibilizado gratuitamente pela Organização Mundial de Saúde para pesquisas na área de saúde e foram analisados com - programa Statistical Package for Social Sciences $\left(\right.$ SPSS $\left.^{\circledR}\right)$, na versão 21.0 .

Este artigo foi aprovado pelo Comitê de Ética em Pesquisa (CEP) da Universidade Federal de Pernambuco (UFPE) sob o parecer de CAAE: 51843715.2.0000.5208, sendo parte integrante do Programa de Iniciação Científica (PIC) da dissertação de mestrado da pesquisadora principal e orientadora deste artigo.

\section{Resultados}

Os resultados e a discussão deste estudo referem-se às entrevistas dos pacientes com tentativa de suicídio no ano de 2015 . Compuseram a população e a amostra do estudo 90 pacientes cujos nomes constaram em notificações compulsórias enviadas por profissionais de saúde para a Vigilância de Atenção a Doenças não Transmissíveis da Secretaria Municipal de Saúde do Recife.

A Tabela 1 caracteriza os pacientes que tentaram suicídio em Recife, nos anos de 2014 a 2015, quanto às variáveis sociais e ocupacionais, e houve predominância de $77,5 \%$ do sexo feminino.
Tabela 1 - Caracterização social e econômica dos pacientes que tentaram suicídio na região metropolitana do Recife no período de novembro de 2014 a outubro de 2015. Recife, PE, Brasil, 2015

\begin{tabular}{|c|c|c|}
\hline Variáveis & n 90 & $\%$ \\
\hline \multicolumn{3}{|l|}{ Idade } \\
\hline $19-28$ & 23 & 29,5 \\
\hline $29-38$ & 34 & 35,9 \\
\hline $39-48$ & 14 & 14,1 \\
\hline $49-58$ & 13 & 12,8 \\
\hline$>59$ & 06 & 7,7 \\
\hline \multicolumn{3}{|l|}{ Sexo } \\
\hline Masculino & 21 & 22,5 \\
\hline Feminino & 69 & 77,5 \\
\hline \multicolumn{3}{|l|}{ Escolaridade } \\
\hline No máximo $1^{\circ} \mathrm{grau}$ & 42 & 46,3 \\
\hline $2^{\circ} \mathrm{grau}$ & 41 & 45,0 \\
\hline Ensino Superior & 07 & 8,7 \\
\hline \multicolumn{3}{|l|}{ Cor da pele } \\
\hline Branca & 25 & 31,25 \\
\hline Preta & 08 & 10,00 \\
\hline Parda & 56 & 57,50 \\
\hline Amarela & 01 & 1,25 \\
\hline \multicolumn{3}{|l|}{ Estado Civil } \\
\hline Solteiro/nunca se casou & 51 & 51,25 \\
\hline Casado ou união estável & 23 & 28,75 \\
\hline Viúvo & 04 & 5,00 \\
\hline Divorciado/separado & 12 & 15,00 \\
\hline \multicolumn{3}{|l|}{ Ocupação } \\
\hline Aposentado(a) & 08 & 10,25 \\
\hline Desempregado(a) & 30 & 35,13 \\
\hline Do lar & 23 & 21,13 \\
\hline Estudante & 11 & 15,04 \\
\hline Emprego informal & 18 & 18,45 \\
\hline \multicolumn{3}{|l|}{ Classe social } \\
\hline $\mathrm{A}^{*}-\mathrm{B}^{+}$ & 21 & 23,1 \\
\hline$C^{\ddagger}$ & 41 & 41,0 \\
\hline$D^{\S}-E^{\|}$ & 28 & 35,9 \\
\hline
\end{tabular}

${ }^{*} \mathrm{R} \$=20.888,00 ;{ }^{\dagger} \mathrm{R} \$: 9.254,00-4.852 ;{ }^{\ddagger} \mathrm{R} \$: 2.705,00-1.625,00 ;$ ${ }^{\S} \mathrm{R} \$=768,00 ; " \mathrm{R} \$ 768,00$

O Distrito Sanitário IV é formado atualmente por 12 bairros, com total de 267.462 habitantes, criado em julho de 1995, como parte do processo de descentralização dos serviços de saúde no âmbito do município com o objetivo de articular ações e serviços de saúde entre os diversos níveis de atenção.

A Figura 1 demonstra o geoprocessamento e os métodos mais utilizados nas tentativas de suicídio na região metropolitana do Recife, no ano de 2015, onde o Distrito Sanitário IV apresenta o maior número dessas tentativas. 


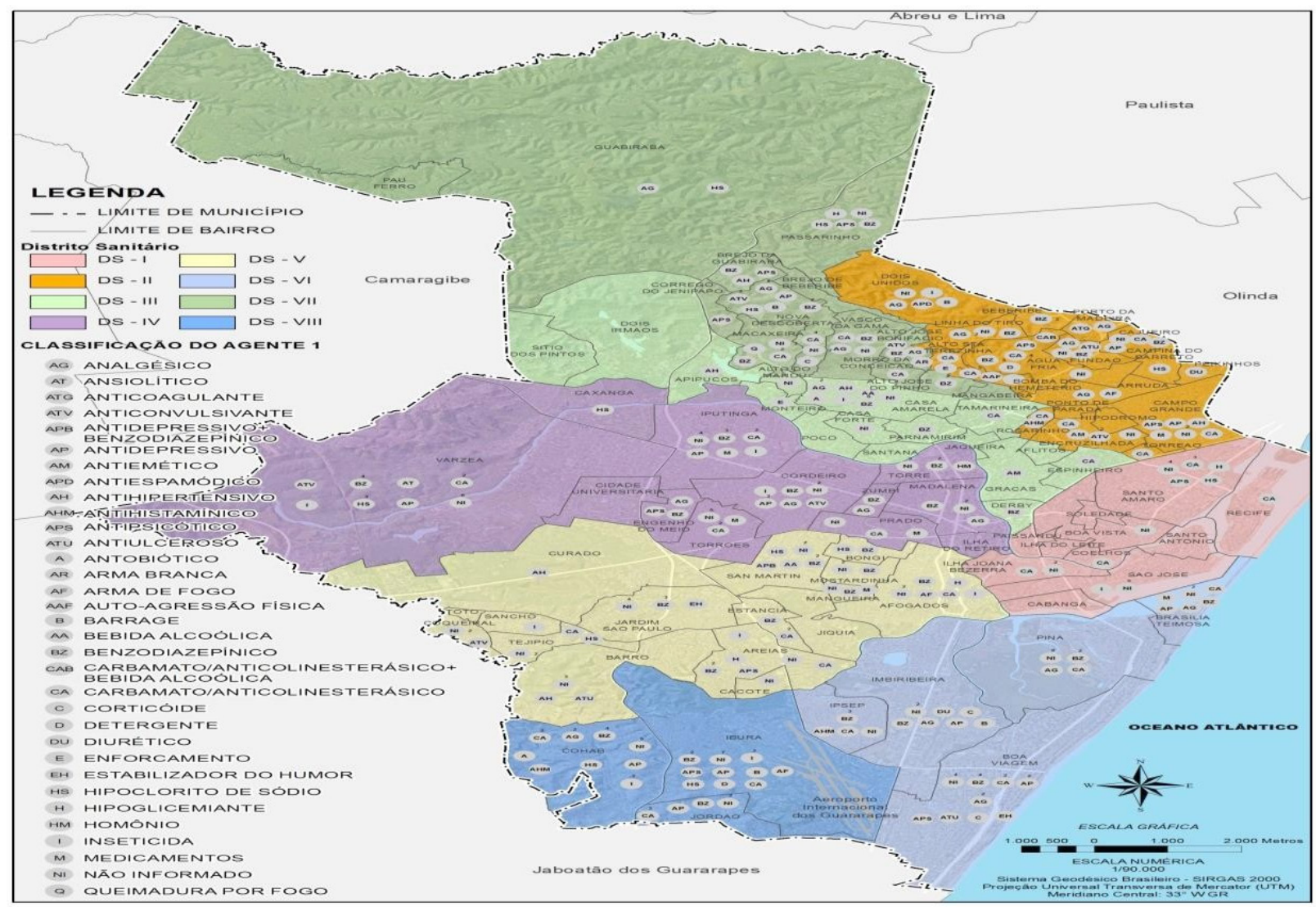

Figura 1 - Geoprocessamento e métodos mais utilizados das tentativas de suicídio na Região Metropolitana do Recife no ano de 2015. Recife, PE, Brasil, 2015

Dentro do geoprocessamento, o Distrito Sanitário IV realizou $18 \%$ do total das notificações referentes às tentativas de suicídio, afirmando o compromisso da obrigatoriedade das notificações compulsórias. Isto não significa que, neste distrito, haja mais tentativas de suicídio do que nos demais decorrentes das subnotificações.

Com relação ao consumo de bebidas alcoólicas, a Tabela 2 registra que cerca de $45 \%$ dos entrevistados nunca consumiram bebida alcoólica; $15 \%$ já o fizeram, porém, não bebem mais e $40 \%$ fazem o uso. Das pessoas que consomem bebida alcoólica, atualmente, $75 \%$ afirmaram que a bebida preferida é a cerveja. Entre as pessoas que afirmaram já ter bebido no passado e que não o fazem atualmente, $16,7 \%$ referiram interrupção do uso por serem alcoolistas. Quanto à idade de início de ingestão, entre os pacientes que bebem e os que já beberam, 52,5\% relataram que tinham entre 15 e 20 anos e 20,5\% afirmaram que a bebida alcoólica já causou problemas familiares. Dos pacientes que afirmaram nunca ter bebido, 25\% justificaram a razão de não beber por não apreciarem o gosto.

Tabela 2 - Distribuição das características relacionadas ao consumo de álcool de pacientes que tentaram o suicídio na região metropolitana do Recife no período de novembro de 2014 a outubro de 2015. Recife, PE, Brasil, 2015

\begin{tabular}{|c|c|c|c|}
\hline Características & Categorias & Frequência & Percentual \\
\hline \multirow[t]{3}{*}{ Consumo de bebidas } & Nunca bebeu & 40 & 45,0 \\
\hline & Já bebeu, mas não bebe mais & 15 & 15,0 \\
\hline & Ainda bebe & 35 & 40,0 \\
\hline \multirow[t]{4}{*}{ Bebida alcoólica preferida* } & Cerveja & 27 & 75,0 \\
\hline & Cachaça & 03 & 9,4 \\
\hline & Uísque & 02 & 6,2 \\
\hline & Outros & 03 & 9,4 \\
\hline \multirow[t]{3}{*}{ Razão de não beber mais ${ }^{\dagger}$} & Não aprecia o gosto & 03 & 25,0 \\
\hline & Já foi alcoolista & 02 & 16,7 \\
\hline & Não teve vontade & 02 & 16,7 \\
\hline
\end{tabular}


Tabela 2 - continuação

\begin{tabular}{|c|c|c|c|}
\hline Características & Categorias & Frequência & Percentual \\
\hline & Outro motivo & 08 & 66,7 \\
\hline \multirow[t]{2}{*}{ Meses sem ingerir bebida alcoólica ${ }^{\dagger}$} & Menor ou igual a um ano & 10 & 79,0 \\
\hline & Mais de um ano & 05 & 21,0 \\
\hline \multirow[t]{3}{*}{ Idade em que começou a beber mais que um gole ${ }^{\ddagger}$} & De 10 a 14 anos & 11 & 39,5 \\
\hline & De 15 a 20 anos & 31 & 52,5 \\
\hline & Maior de 20 anos & 05 & 8,0 \\
\hline \multirow[t]{4}{*}{ Problemas causados pela bebida ${ }^{\ddagger}$} & Problemas com a família & 20 & 45,0 \\
\hline & Problemas de saúde & 12 & 12,0 \\
\hline & Problemas no trabalho & 15 & 33,5 \\
\hline & Problemas com a lei ou Polícia & 03 & 9,5 \\
\hline \multirow[t]{6}{*}{ Razão pra não beber§ } & Não aprecia o gosto & 15 & 40,4 \\
\hline & Não teve vontade & 08 & 12,0 \\
\hline & Religião & 09 & 19,7 \\
\hline & Criação & 03 & 10,0 \\
\hline & Saúde/Uso de medicamentos & 03 & 10,0 \\
\hline & Outro motivo & 02 & 9,9 \\
\hline
\end{tabular}

* Respondida por quem ainda bebe; ${ }^{+}$Respondida por quem já bebeu, mas não bebe mais;

${ }^{\ddagger}$ Respondida por quem bebe ou já bebeu; ${ }^{\S}$ Respondida por quem nunca bebeu

Dentre os pacientes que afirmaram ainda beber, apenas $9,4 \%$ (três pacientes) relataram que precisam beber mais que antes para o álcool ter o mesmo efeito. Além disso, cerca de $54,8 \%$ dos pacientes usuários de bebidas alcoólicas nunca consumiram vinho, 7,8\% nunca consumiram cerveja e 35,5\% nunca consumiram bebida destilada, como pode ser observado na Tabela 3. Dentre os que tomam cerveja, $29,5 \%$ bebem, no mínimo, uma vez por semana e 20,6 tomam mais de 12 doses de cerveja em dia comum.

Tabela 3 - Distribuição das características relacionadas à frequência do consumo de álcool de pacientes que tentaram na região metropolitana do Recife no período de novembro de 2014 a outubro de 2015. Recife, PE, Brasil, 2015

\begin{tabular}{|c|c|c|c|}
\hline Características & Categorias & Frequência & Percentual \\
\hline \multirow[t]{4}{*}{ Frequência na qual bebeu vinho nos últimos 12 meses } & Nunca & 21 & 54,8 \\
\hline & Menos de uma vez por mês & 09 & 29,0 \\
\hline & Uma a três vezes no mês & 02 & 6,5 \\
\hline & Ao menos, uma vez na semana & 03 & 9,7 \\
\hline \multirow[t]{3}{*}{ Doses de vinho em dia comum } & De uma a duas doses & 05 & 33,3 \\
\hline & De três a quatro doses & 07 & 54,7 \\
\hline & Mais do que quatro doses & 02 & 12,0 \\
\hline \multirow[t]{7}{*}{ Frequência na qual bebeu cerveja nos últimos 12 meses } & Nunca & 02 & 7,8 \\
\hline & Menos de uma vez por mês & 03 & 9,2 \\
\hline & Uma a três vezes no mês & 08 & 20,5 \\
\hline & Uma a duas vezes por semana & 11 & 29,5 \\
\hline & Três a quatro vezes por semana & 09 & 25,5 \\
\hline & Cinco a seis vezes por semana & 02 & 7,5 \\
\hline & Todos os dias & 02 & 6,5 \\
\hline \multirow[t]{5}{*}{ Doses de cerveja em dia comum } & Até quatro doses & 05 & 19,7 \\
\hline & De cinco a oito doses & 19 & 40,1 \\
\hline & De nove a doze doses & 03 & 13,1 \\
\hline & Mais de doze doses & 06 & 20,6 \\
\hline & Nunca & 14 & 35,5 \\
\hline \multirow[t]{3}{*}{ Frequência na qual bebeu destilado nos últimos 12 meses } & Menos de uma vez por mês & 11 & 32,3 \\
\hline & Uma a três vezes no mês & 05 & 16,1 \\
\hline & Ao menos, uma vez na semana & 05 & 16,1 \\
\hline
\end{tabular}


Com relação aos pacientes entrevistados que tentaram suicídio em Recife, observa-se, na Tabela 4, que cerca de $41,4 \%$ dos entrevistados encontram-se na Zona I, que requer aconselhamento quanto aos riscos do consumo de álcool.

$\mathrm{Na} Z$ Zona II, formada por $24,1 \%$ dos pacientes que consomem bebida alcoólica, faz-se necessário aconselhamento frequente para esse grupo de pacientes. Já a Zona IV, formada por $27,6 \%$ dos pacientes que bebem, mostra que estes precisam de um referenciamento a um especialista para tratamento contra o alcoolismo.

Tabela 4 - Teste AUDIT dos pacientes que tentaram o suicídio na região metropolitana do Recife no período de novembro de 2014 a outubro de 2015. Recife, PE, Brasil, 2015

\begin{tabular}{ccc}
\hline $\begin{array}{c}\text { Teste AUDIT } \\
\text { Frequência } \\
\text { Percentual }\end{array}$ & $\begin{array}{c}\text { Teste AUDIT } \\
\text { Frequência } \\
\text { Percentual }\end{array}$ & $\begin{array}{c}\text { Teste AUDIT } \\
\text { Frequência } \\
\text { Percentual }\end{array}$ \\
\hline Zona I & 15 & 41,4 \\
Zona II & 08 & 24,1 \\
Zona III & 02 & 6,9 \\
Zona IV & 10 & 27,6 \\
Total & 35 & 100,0 \\
\hline
\end{tabular}

\section{Discussão}

As mulheres apresentam uma frequência de tentativa de suicídio três vezes superior à dos homens, porém, a não prevalência deste gênero nos suicídios consumados deve-se à maior procura aos serviços de saúde, ao engajamento com a religiosidade e/ou espiritualidade, baixa prevalência de alcoolismo e maior flexibilidade com os papéis e aptidões sociais(7).

Em contrapartida, os homens apresentam uma incidência maior em relação ao suicídio consumado, utilizando, para tal, mecanismos letais. As maneiras mais utilizadas para cometer o suicídio, segundo estudos no mundo inteiro, são o enforcamento, a intoxicação e as armas de fogo ${ }^{(8)}$.

Quanto ao fator idade, 35,9\% dos entrevistados tinham entre 29 a 38 anos quando realizaram a tentativa de suicídio. O Brasil encontra-se em 670 lugar no ranking mundial no que se refere ao suicídio ${ }^{(9)}$ e entre as dez principais causas de morte para todas as pessoas acima dos cinco anos de idade, enquadrando-se entre as três principais causas de morte entre as idades de 15 e 44 anos. Essas mudanças da fase da adolescência para a adulta carregam consigo comportamentos de riscos, principalmente em atividades que comprometem a saúde física e mental, assim como a influência do meio, trazendo consequências nos níveis individual, familiar e social(10).
Tanto as tentativas quanto o suicídio vêm aumentando assustadoramente entre a população jovem nas últimas décadas, fazendo com que eles ocupem o grupo de maior risco, ficando com a segunda principal causa de morte entre os indivíduos de dez a 24 anos de idade(8).

Em relação à escolaridade, $46,3 \%$ dos pesquisados apresentam, no máximo, o primeiro grau do Ensino Fundamental. Este baixo nível de escolaridade é bastante comum entre os que tentam suicídio.

Dentre a população pesquisada, $57,50 \%$ autodeclaram-se de cor de pele parda. As pessoas que apresentam raça/cor parda têm uma maior tendência ao suicídio. Essas características, tanto culturais quanto étnicas, podem ser fatores que influenciam o crescente número das tentativas e óbitos, podendo ser explicáveis por questões etnológicas e psiquiátricas ${ }^{(11)}$.

De acordo com os pesquisados, $51,25 \%$ dos que tentaram suicídio apresentavam como estado civil ser solteiros, traçando um grupo da população mais acometida pelo evento.

Estudos recentes apontam uma correlação às tentativas de suicídio entre os indivíduos solteiros e casados no que se refere às situações e vivências que cada um tem, particularmente. Uma pesquisa realizada no Sul do Brasil apontou que mulheres casadas apresentam maiores chances de cometer suicídio(12).

Quanto às pessoas que cometeram suicídio e suas ocupações/profissões, os dados coletados revelam que $35,13 \%$ estavam desempregados, $21,13 \%$ afirmaram desenvolver atividades domésticas em suas residências e, em sequência, $18,45 \%$ possuíam empregos informais, $15,04 \%$ eram estudantes e $10,25 \%$, aposentados.

Outro grande fator para o elevado número de reincidência das tentativas de suicídio é o desemprego. Estudiosos afirmam que as grandes crises econômicas geram altas taxas de desemprego e podem fomentar sentimentos de desesperança seguidos por depressão e pensamentos suicidas(13).

A classe social C ( $R \$: 2.705,00-1.625,00$ reais) representa $41 \%$ do quantitativo das tentativas de suicídio dos indivíduos entrevistados, sendo $A$ ( $R$ \$: $20.888,00$ reais) e $B(R \$: 9.254,00-4.852,00), 23,11 \%$ e $D(R \$: 768,00$ reais) - E ( $R$ \$: 768,00 reais), 35,9\%.

De acordo com a OMS, o poder aquisitivo da população, quando polarizado (alta e baixa renda), torna-se fator que gera riscos para o suicídio e a maioria dos indivíduos que tentaram suicídio pertencia às classes $C$ ( $R \$: 2.705,00-1.625,00$ reais), $D(R \$: 768,00$ reais) e $E(R \$: 768,00 \text { reais })^{(14)}$.

Sobre os métodos mais utilizados, $44,04 \%$ ficaram com a dose aumentada de medicamentos, pois, mesmo essas drogas sendo vendidas por meio de receitas médicas, o livre acesso a elas ainda é comum. 
Em alguns casos, os indivíduos já fazem uso crônico. Outros meios para tentativa são utilizados, como arma de fogo e enforcamento, fazendo-se necessárias campanhas frequentes sobre a utilização dos mesmos nos suicídios ou nas tentativas ${ }^{(8)}$.

O consumo de bebidas alcoólicas é uma prática corrente em todo mundo, causando frequentes e inúmeros problemas clínicos, psicológicos, profissionais e familiares. No Brasil, este problema acentua-se, pois esta droga é vendida abertamente, sem que haja algum tipo de fiscalização, além das diversas propagandas de incentivo para o consumo nos diversos tipos de veículo de comunicação(15-16).

O consumo de drogas ilícitas tem se tornado cada vez mais precoce, com o primeiro contato logo na infância, sendo o mais frequente o álcool. O abandono, a negligência, a falta de diálogo familiar, a agressão física e a cultura do uso de drogas são possíveis determinantes para a iniciação ao abuso durante a juventude(16).

O abuso e a dependência destas substâncias tornam-se um grande problema de saúde pública no mundo e no Brasil. Em 2012, o segundo Levantamento Nacional de Álcool e Drogas revelou que cerca de 67 milhões de pessoas consomem álcool regularmente e 11 milhões apresentam uso abusivo ou dependência(17).

Estudos realizados em um Centro de Atenção Psicossocial traçaram o uso de drogas como a principal motivação para o pensamento suicida. Relações familiares fragilizadas e a desesperança tornam-se fator primordial para a intenção de eliminar a própria vida como modo de cessar o incômodo familiar(18).

A intoxicação alcoólica que antecede as tentativas de suicídios dos chamados alcoólatras deprimidos apresenta um ápice no consumo de álcool na véspera do evento. Trata-se de uma relação dose-resposta na qual quanto maior o consumo de álcool maior é a chance de comportamento suicida(19).

Alcoólatras apresentam 60 a 120 vezes mais probabilidade de cometer o suicídio do que a população abstêmia. Em vários países, a diminuição da ingestão de bebidas que contêm álcool fez despencar as incidências dos eventos de suicídio(20). Esses achados afirmam os dados já conhecidos pelas diversas literaturas de que as tentativas de suicídio ocorrem, com maior frequência, entre estes indivíduos que sofrem dos transtornos relacionados ao uso de bebidas alcoólicas(20).

Percebe-se, considerando os agravos dos problemas relacionados ao alcoolismo e sua relação com as taxas de suicídio, que não existe uma quantidade segura quando se trata da ingestão de substâncias alcoólicas. Há um aumento gradativo do consumo destas e alguns indivíduos, mesmo que ainda não alcoólatras, podem ter problemas sociais, familiares, falta de emprego, acidentes e, em alguns casos, suicídio(21).

O uso crônico e excessivo do álcool produz efeitos de caráter depressor, estimulante e euforizante no sistema nervoso central, sofrendo desaprovação social e a negação da dependência por parte do usuário(22). Como visto, o teste AUDIT caracteriza a presença de problemas atribuíveis ao uso excessivo de álcool.

Quanto aos resultados do teste AUDIT, é importante ressaltar que os enfermeiros, em seu papel de promotores de saúde e detentores de práxis voltada para essas demandas nos serviços de saúde, possuem uma grande responsabilidade na identificação das situações de riscos, sobretudo ao abuso e à dependência que podem acarretar comportamentos suicidas ${ }^{(5)}$.

Quanto mais informações adquiridas pela equipe multidisciplinar de saúde, melhor será o planejamento para uma abordagem terapêutica adequada, ressaltando que cada indivíduo é diferente do outro e que o álcool atua de modo negativo no controle, na motivação, na atitude interna e contribui materialmente para o impulso do comportamento suicida(23).

\section{Conclusão}

O estudo possibilitou traçar o perfil socioeconômico dos/das usuários/as que tentaram suicídio nos oito DS do município do Recife, no ano de 2015. Observou-se uma prevalência maior em mulheres jovens, com idade média de 38 anos, com primeiro grau de escolaridade, cor parda, solteiras, desempregadas e pertencentes à classe social C ( $\$ \$$ : $2.705,00-1.625,00$ reais).

Vale salientar que os comportamentos suicidas se acentuam com o uso abusivo e contínuo do álcool, fazendo com que aumentem, desenfreadamente, os números, tanto dos suicídios quanto das tentativas. Dessa forma, é preciso compreender as causas desencadeadoras e as suas consequências para que se possa intervir, de maneira eficaz, pois falar sobre o suicídio, seus desencadeadores e suas consequências ainda é a forma mais eficaz para combater este problema de saúde mundial.

O estudo também evidenciou que o abuso do álcool tem direta relação com o livre acesso ao mesmo, sendo a cerveja a droga mais utilizada. Apesar da legislação brasileira proibir sua comercialização a menores de 18 anos de idade, o contato com esta droga tem se tornado cada vez mais precoce e mais frequente, tanto por estímulos familiares, como midiáticos.

Esta pesquisa demonstra, ainda, que o uso contínuo do álcool traz diversos problemas com repercussões individuais e coletivas, principalmente, em relação ao ambiente familiar. Os problemas mais frequentes com o uso do álcool são os conflitos familiares, a falta de emprego, acidentes de trânsito e agravos à saúde 
decorrentes do uso crônico, resultando, por vezes, em tentativas de suicídio.

Assim, a difusão de estratégias para combater o uso de drogas, em especial, o álcool, e o suicídio, sensibilizando e esclarecendo a população, associada a uma assistência humanizada e ao acompanhamento multidisciplinar na atenção básica, é de extrema importância e capaz de identificar, diagnosticar e tratar possíveis comportamentos suicidas das comunidades adstritas.

Nesta perspectiva, é preciso que o tema tentativa de suicídio, com enfoque ao uso de álcool e outras drogas, seja discutido e encarado com seriedade pelas equipes de saúde, enfatizando o respeito e o compromisso com esses indivíduos. É preciso, também, que haja planos de capacitações para os profissionais de saúde, proporcionando o desenvolvimento de habilidades necessárias à identificação destes perfis, visando a auxiliar com processos interventivos aos indivíduos que tenham tentado quanto à sua autodestruição.

Além dos esclarecimentos trazidos sobre a relação do uso do álcool e o suicídio, este estudo apresentou limitações na sua execução. Uma limitação foi a ausência de notificações de todos os casos de tentativa de suicídio, ocasionando incógnitas quanto ao real número de pessoas com tentativa de suicídio na região estudada. E outra foi tocante aos casos notificados com preenchimento de dados pessoais dos indivíduos e à mudança de endereço durante o estudo, impossibilitando a coleta de alguns casos.

Embora esta pesquisa tenha apresentado limitação na execução, ela contribui para o entendimento sobre a acentuação dos comportamentos suicidas com o uso abusivo do álcool e, por isso, este problema de saúde pública mundial deve ser encarado pelas equipes de saúde com seriedade, respeito e compromisso, pois os agravos e as consequências dessas complicações se tornam ainda mais difíceis quando negligenciados pelos profissionais de saúde.

\section{Referências}

1. Ferreira NS, Pessoa VF, Barros AB, Figueiredo AEB, Minayo MCS. Fatores de risco relacionados com suicídio em Palmas (TO), Brasil, 2006-2009, investigados por meio de autópsia psicossocial. Ciênc Saúde Coletiva. [Internet]. 2014 fev 19(1):115-26. doi: 10.1590/1413-81232014191.2229

2. Ministério da Saúde. Novos dados reforçam a importância da prevenção do suicídio [Acesso 16 out 2018]. Disponível em: < http://portalms.saude.gov.br/noticias/agenciasaude/44404-novos-dados-reforcam-a-importancia-daprevencao-do-suicidio $>$.
3. Meleiro A, Teng CT, Wang YP. Suicídio: estudos fundamentais. Rev Bras Psiquiatr. 2005 set 27(3). doi: 10.1590/S1516-44462005000300025

4. Carlini EA, Rodrigues E, Mendes FR, Tabach R, Gianfratti B. Treatment of drug dependence with Brazilian herbal medicines. Rev Bras Farmacogn. 2006 Dec 16(supl.):690-5. doi: 10.1590/ S0102-695X2006000500016

5. Ministério da Saúde (BR). Prevenção do Suicídio: Manual dirigido a profissionais das equipes de saúde mental. 2006, 56. Disponível em: <https://www.nescon. medicina.ufmg.br/biblioteca/imagem/1241.pdf>.

6. World Health Organization. The Alcohol Use Disorders Identification Test. Geneva: WHO; 2001. Available from: <https://apps.who.int/iris/bitstream/ handle/10665/67205/WHO_MSD_MSB_01.6a.pdf;jsess ionid $=521 \mathrm{F0D} 3 \mathrm{D} 090 \mathrm{EC} 74 \mathrm{CB} 9 \mathrm{~B} 416 \mathrm{FEB} 717 \mathrm{D} 835$ ? seque nce $=1>$.

7. Braga LL, Aglio DDD. Suicide in adolescence: risk factors, depression and gender. Clin Contexts. 2013 Jan./June. 6(1):2-14. Avaliable from: <http://revistas. unisinos.br/index.php/contextosclinicos/article/view/ ctc. 2013.61.01/1533>.

8. Soares RJO, Nascimento FPB. Suicide and suicide attempt: Construction of Brazilian Nursing. J Health Sci. 2017;19(1):19-24. doi: 10.17921/2447-8938.2017v19 n1p19-24

9. Alves VM, Francisco LCFL, Belo FMP, De-Melo-Neto VL, Barros VG, Nardi AE. Evaluation of the quality of life and risk of suicide. Clinics. 2016 Mar 71(3):135-9 doi: $10.6061 /$ clinics/2016(03)03

10. Botega NJ. Suicidal behavior: Epidemiology. Psicol. USP. 2014 Sept./Dec. 25(3): 231-6. doi: 10.1590/0103-6564D20140004

11. Pedrosa NFC, Barreira DA, Rocha DQC, Barreira MA. Análise dos principais fatores epidemiológicos relacionados ao suicídio em uma cidade no interior do Ceará, Brasil. J Health Biol Sci. 2018; 6(4):399-404. doi: 10.12662/2317-3076jhbs.v6i4.2068.p399-404.2018 12. Ferreira VRT, Trichês VJS. Epidemiological profile of suicide attempts and deaths in a city in the South of Brazil. Psico 2014 Apr./June 45(2):219-27. Avaliable from: <http://revistaseletronicas.pucrs.br/ojs/index. php/revistapsico/article/view/13980/11711>.

13. Chan CH, Caine ED, You S, Fu KW, Chang SS, Yip PS. Suicide rates among working-age adults in South Korea before and after the 2008 economic crisis. J Epidemiol Commun Health 2014; 68:246-52. doi: 10.1136/ jech-2013-202759

14. World Health Organization. Preventing suicide: A global imperative. Geneva: WHO; 2015. p. 123. . Available from: <https://apps.who.int/iris/bitstream/ handle/10665/131056/9789241564779_eng. pdf?sequence $=1>$. 
15. Dázio EMR, Zago MMF, Fava SMCL. Use of alcohol and other drugs among male college students and their meanings. Rev Esc Enferm USP. 2016;50(5):785-91. doi: 10.1590/s0080-623420160000600011

16. Vieira DL, Ribeiro M, Laranjeira R. Evidence of association between early alcohol use ans risk of later problems. Braz J Psychiatry. 2007 Sept 29(3):222-7. doi: 10.1590/S1516-44462007000300006

17. LENAD. II National Survey of Alcohol and Drugs: National Institute of Science and Technology for Public Policies of Alcohol and Other Drugs. UNIFESP 2012; 04:54. 18. Miranda R, Ortin A, Scott M, Shaffer D. Characteristics of suicidal ideation that predict the transition to future suicide attempts in adolescents. J Child Psychol Psychiatry. 2014 Nov 55(11):1288-96. doi: 10.1111/ jcpp. 12245

19. Fontenelle P. Suicide - The Future Interrupted: Guide to Survivors. Geração editorial; 2008.

20. Gupta A, Priya B, Williams J, Sharma M, Gupta R, Jha DK, et al. Intra-household evaluations of alcohol abuse in men with depression and suicide in women: A cross-sectional community-based study in Chennai, India. BMC Public Health 2015;15:636. doi: 10.1186/ s12889-015-1864-5

21. Laranjeira, R. Users of Psychoactive Substances. CREMESP 2003; 09:65.

22. Gigliotti A, Bessa MA. Alcohol Dependence Syndrome: diagnostic criteria. Braz J Psychiatry. 2004; 26 (supl 1):11-3. doi: 10.1590/S1516-44462004000500004. 23. Duncan BB. Ambulatory Medicine: Evidence Based Primary Care. 2012;3:3.

\section{Contribuição dos autores}

Concepção e planejamento do estudo: Eliana Lessa Cordeiro, Liniker Scolfild Rodrigues da Silva, Emmanuel Wagner Pereira Mendes e Luiz Claudio Luna da Silva. Obtenção dos dados: Emmanuel Wagner Pereira Mendes, Luiz Claudio Luna da Silva e Vanessa Lacerda Duarte. Análise e interpretação dos dados: Emmanuel Wagner Pereira Mendes, Vanessa Lacerda Duarte e Liniker Scolfild Rodrigues da Silva. Redação do manuscrito: Luiz Claudio Luna da Silva, Vanessa Lacerda Duarte, Évelyn Cristina Morais Pessôa Lima e Liniker Scolfild Rodrigues da Silva. Revisão crítica do manuscrito: Eliana Lessa Cordeiro, Liniker Scolfild Rodrigues da Silva e Évelyn Cristina Morais Pessôa Lima.

Todos os autores aprovaram a versão final do texto.
Conflito de interesse: Os autores declaram não haver conflito de interesse.
Recebido: 16.04.2019

Aceito: 27.08 .2019
Copyright $\odot 2020$ SMAD, Rev. Eletrônica Saúde Mental Álcool Drog. Este é um artigo de acesso aberto distribuído sob os termos da Licença Creative Commons CC BY-NC.

Esta licença permite que outros remixem, adaptem e criem a partir do seu trabalho para fins não comerciais, e embora os novos trabalhos tenham de lhe atribuir o devido crédito e não possam ser usados para fins comerciais, os usuários não têm de licenciar esses trabalhos derivados sob os mesmos termos. 